\title{
Exertional dyspnoea and cortical oxygenation in patients with COPD
}

\author{
Yuji Higashimoto ${ }^{1}$, Noritsugu Honda ${ }^{2}$, Toshiyuki Yamagata ${ }^{1}$, Akiko Sano ${ }^{1}$ \\ Osamu Nishiyama ${ }^{1}$, Hiroyuki Sano', Takashi Iwanaga', Hiroaki Kume ${ }^{1}$, \\ Yasutaka Chiba ${ }^{3}$, Kanji Fukuda ${ }^{2}$ and Yuji Tohda ${ }^{1}$
}

Affiliations: ${ }^{1}$ Dept of Respiratory Medicine and Allergology, Kinki University, Osaka, Japan. ${ }^{2}$ Dept of Rehabilitation Medicine, Kinki University, Osaka, Japan. ${ }^{3}$ Division of Biostatistics, Clinical Research Center, Kinki University, Osaka, Japan.

Correspondence: Yuji Higashimoto, Dept of Respiratory Medicine and Allergology, Kinki University, Faculty of Medicine, 377-2 Ohnohigashi, Osakasayama, Osaka 589-8511, Japan. E-mail: yhigashiđmed.kindai.ac.jp

ABSTRACT This study was designed to investigate the association of perceived dyspnoea intensity with cortical oxygenation and cortical activation during exercise in patients with chronic obstructive pulmonary disease (COPD) and exertional hypoxaemia.

Low-intensity exercise was performed at a constant work rate by patients with COPD and exertional hypoxaemia $(n=11)$ or no hypoxaemia $(n=16)$, and in control participants $(n=11)$. Cortical oxyhaemoglobin $(\mathrm{oxy}-\mathrm{Hb})$ and deoxyhaemoglobin (deoxy-Hb) concentrations were measured by multichannel near-infrared spectroscopy. Increased deoxy- $\mathrm{Hb}$ is assumed to reflect impaired oxygenation, whereas decreased deoxy- $\mathrm{Hb}$ signifies cortical activation.

Exercise decreased cortical deoxy-Hb in control and nonhypoxaemic patients. Deoxy-Hb was increased in hypoxaemic patients and oxygen supplementation improved cortical oxygenation. Decreased deoxy-Hb in the pre-motor cortex (PMA) was significantly correlated with exertional dyspnoea in control participants and patients with COPD without hypoxaemia. In contrast, increased cortical deoxy-Hb concentration was correlated with dyspnoea in patients with COPD and hypoxaemia. With the administration of oxygen supplementation, exertional dyspnoea was correlated with decreased deoxy-Hb in the PMA of COPD patients with hypoxaemia.

During exercise, cortical oxygenation was impaired in patients with COPD and hypoxaemia compared with control and nonhypoxaemic patients; this difference was ameliorated with oxygen supplementation. Exertional dyspnoea was related to activation of the pre-motor cortex in COPD patients.

@ERSpublications

Exertional dyspnoea was related to activation of the pre-motor cortex in COPD patients http://ow.ly/QUHfC

For editorial comments see Eur Respir J 2015; 46: 1554-1556 [DOI: 10.1183/09031936.01450-2015]

This article has supplementary material available from erj.ersjournals.com

Received: Sept 012014 | Accepted after revision: July 082015 | First published online: Oct 222015

Conflict of interest: Disclosures can be found alongside the online version of this article at erj.ersjournals.com

Support statement: This study was funded by the Japan Society for the Promotion of Science (grant 26461171). Funding information for this article has been deposited with FundRef.

Copyright OERS 2015 


\section{Introduction}

Chronic obstructive pulmonary disease (COPD) is a leading cause of mortality worldwide. Some patients with COPD have exertional hypoxaemia without resting hypoxaemia and are not candidates for home oxygen therapy [1, 2]. Although some previous reports have shown that ambulatory oxygen supplementation does not provide benefits for dyspnoea and the short-term quality of life of these patients [3], a meta-analysis found that oxygen use relieves breathlessness [4]. Furthermore, impaired cortical oxygenation may affect the cognitive functioning of patients [5]. However, there is little information on the cortical oxygenation and haemodynamics of patients with COPD [6, 7]. JENSEN et al. [6] showed that cerebral oxygenation was impaired in terminal lung disease, including COPD, and was improved by oxygen supplementation. Most patients with COPD complain about exertional dyspnoea, regardless of exertional hypoxaemia, but the neurophysiology of dyspnoea associated with COPD has yet to be well evaluated $[8,9]$. Recent reports, using functional magnetic resonance imaging (fMRI), show that activation of the limbic system, amygdala and anterior insula is essential for the perception of dyspnoea in healthy volunteers $[8,10,11]$; however, the association of cerebral activation with the perception of dyspnoea has not been studied in patients with COPD. Based on the multichannel near-infrared spectroscopy (NIRS) technique, we previously reported that the pre-frontal cortex was activated in association with exertional dyspnoea in patients with COPD [12]. Despite the ability of fMRI to measure activation of the entire brain at high spatial resolution, it is relatively difficult to examine cerebral haemodynamics during exercise using this technique. In contrast, NIRS is an established method for measuring cortical haemodynamics during exercise $[13,14]$. To the best of our knowledge, there is no report on the association between cortical haemodynamics and perceived dyspnoea intensity (PDI) in patients with COPD.

We hypothesised that cortical haemodynamics differ between patients with and without exertional hypoxaemia, and that cortical activation (oxygenation) is associated with PDI as well as hypoxaemia. This study is therefore the first to monitor sequential changes in cortical haemodynamics and cortical activation in relation to PDI in patients with COPD and in control participants.

\section{Methods}

\section{Participants}

Twenty-seven right-handed, stable and symptomatic male patients with COPD were recruited for enrolment in this study from patients who visited the Dept of Respiratory Medicine and Allergology at Kinki University Hospital between May 2009 and February 2013. Exclusion criteria included any other unstable medical conditions that could cause or contribute to breathlessness (i.e. metabolic, cardiovascular or other respiratory diseases) or any other disorders that could interfere with exercise testing, such as neuromuscular diseases or musculoskeletal problems. We also recruited 11 age-matched male volunteers who did not have any detectable chronic condition, including pulmonary or cardiovascular disease. The patients with COPD were divided into two groups according to the presence or absence of exertional hypoxaemia $(\mathrm{n}=11$ hypoxaemic or $\mathrm{n}=16$ nonhypoxaemic patients) during constant work rate cycling exercise (CWRE). In this study, hypoxaemia was defined as a $<90 \%$ arterial oxygen saturation measured by pulse oximetry $\left(\mathrm{SpO}_{2}\right)$ or $\geqslant 4 \%$ decrease from baseline saturation during CWRE [1]. All participants provided signed informed consent and the protocol was approved by the Committee for Ethics at Kinki University School of Medicine.

\section{Multichannel NIRS}

We used a multichannel NIRS instrument (Hitachi Medical, Tokyo, Japan) that measured the time course of relative changes in the concentrations of oxyhaemoglobin (oxy- $\mathrm{Hb})$, deoxyhaemoglobin (deoxy- $\mathrm{Hb})$ and total haemoglobin (total-Hb) $[12,15-17]$ (online supplementary figure S1). We placed three arrays, mounted on a flexible cap, over the pre-frontal $(3 \times 5$ arrays with eight incident and seven detection optical fibres) and bilateral parietal regions $(4 \times 4$ arrays with eight incident and eight detection optical fibres) on each hemisphere (figure 1). An increased concentration of oxy- $\mathrm{Hb}$ or decreased concentration of deoxy-Hb was assumed to indicate cortical activation and an increased total-Hb was indicative of an increase in total cortical blood flow [18]. In contrast, decreased oxy-Hb or increased deoxy-Hb indicated impaired oxygenation of the cortex.

Overall, cortical haemodynamics were evaluated by each of the three probes, but for further analysis of cortical activation we analysed eight cortical areas to find the area most implicated in the perception of dyspnoea (figure 1). The pre-frontal probe was targeted near the dorsolateral and orbitofrontal area in the pre-frontal cortex, and the parietal probe was targeted near the parietal cortex and included the primary somatosensory cortex (S1) and the dorsal part of the frontal cortex, including the pre-motor cortex (PMA) and the primary motor cortex (M1) $[19,20]$.

\section{Low-intensity CWRE}

Exercise tests were performed using an electronically braked cycle ergometer (Combi Wellness, Tokyo, Japan) and breath-by-breath gas analysis was completed using indirect calorimetry (Minato Medical 
FIGURE 1 Schema of the multichannel near-infrared spectroscopy probes. PMA: pre-motor cortex; M1: primary motor cortex; S1: primary somatosensory cortex.
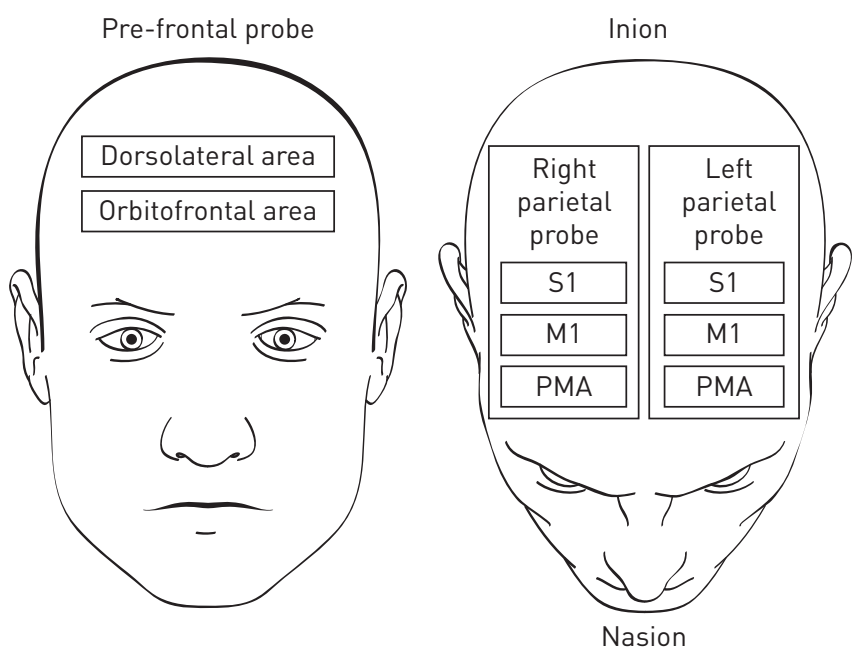

Science, Osaka, Japan) in a temperature-controlled room. During an incremental exercise test, 8 min of low-intensity CWRE was undertaken after a 1-min rest and 1-min warm-up period, with NIRS being recorded at a work intensity corresponding to $40 \%$ of a participant's symptom-limited peak work rate [21]. PDI was determined each minute, during both exercise and rest periods, using the 10-point Borg scale $[22,23]$. Cardiopulmonary and breathing pattern parameters were continuously measured during exercise. Patients with COPD and exertional hypoxaemia received oxygen supplementation ( $3 \mathrm{~L} \cdot \mathrm{min}^{-1}$ ) immediately after the first CWRE and the CWRE was repeated with oxygen supplementation after $10 \mathrm{~min}$ of recovery time.

\section{Statistical analyses}

Data are reported as the mean \pm SEM. A p-value of 0.05 was considered significantly different for all analyses. The data were analysed by comparisons among groups using ANOVA with repeated measures, followed by individual contrasts (Dunnett's method or Tukey's honestly significant difference test). Correlations between the PDI (using Borg scale averages) and the average of measurements obtained during the last $4 \mathrm{~min}$ of exercise were assessed using Spearman rank correlation analysis $(\rho)$. Analyses were performed using JMP 11.2.0 (SAS Institute, Cary, NC).

\section{Results}

\section{Participant characteristics}

Exertional hypoxaemia was detected in 11 patients with COPD in the hypoxaemia group, but not in the 16 patients with COPD in the nonhypoxaemic group or in the control participants. The baseline clinical characteristics of each group are shown in table 1. For all groups, age, gender and body mass index were closely matched. Forced expiratory volume in $1 \mathrm{~s}$ (FEV1), FEV1 \% predicted and peak work load were lower in both the hypoxaemic and nonhypoxaemic COPD groups compared with the control group. All parameters were similar between the hypoxaemic group and nonhypoxaemic group, with the exception of baseline $\mathrm{SpO}_{2}$, which was lower in the hypoxaemic group compared with the other groups.

\section{Prevalence of exertional hypoxaemia and cortical oxygenation during low-intensity CWRE}

Peak PDI was higher in the hypoxaemic group of patients with COPD than in the control participants (4.04 \pm 0.36 versus $2.09 \pm 0.37 ; \mathrm{p}<0.05)$, but was not significantly different from the mean peak PDI of the nonhypoxaemic group $(2.88 \pm 0.44)$. In the hypoxaemic group, the average $\mathrm{SpO}_{2}$ was $<90 \%$ after 5 min of exercise $(88.2 \pm 0.9 \%)$ and lowest after $8 \mathrm{~min}(86 \pm 1.1 \%)$ (figure 2). The $\mathrm{SpO}_{2}$ in this same group was significantly lower than seen in the nonhypoxaemic and control groups at all times during exercise. Oxygen supplementation improved $\mathrm{SpO}_{2}$ in the hypoxaemic group and maintained $\mathrm{SpO}_{2}$ at $>90 \%$ (online supplementary figure S2), and the peak PDI improved in six out of 11 patients; however, the change in peak PDI did not reach statistical significance $(2.15 \pm 0.73)$. Tidal volume $(V \mathrm{~T})$ increased gradually during exercise and reached a similar plateau at $5 \mathrm{~min}$ in all groups. End-tidal carbon dioxide tension $\left(\mathrm{PETCO}_{2}\right)$ was increased during the first 3-4 min, including the warm-up period, and reached a plateau in the control and nonhypoxaemic groups (online supplementary table S1). However, $\mathrm{PETCO}_{2}$ was unchanged in the hypoxaemic group during exercise. $\mathrm{PETCO}_{2}$ was lower in both groups of patients with COPD compared with control participants during exercise, but there was no difference between the hypoxaemic and nonhypoxaemic patients. 


\section{TABLE 1 Participant characteristics at baseline}

Control

COPD

\begin{tabular}{|c|c|c|c|}
\hline & & Without hypoxaemia & With hypoxaemia \\
\hline Participants n & 11 & 16 & 11 \\
\hline Age years & $70.5 \pm 2.4$ & $72.9 \pm 1.7$ & $71.2 \pm 2.1$ \\
\hline Males $n$ & 11 & 16 & 10 \\
\hline \multicolumn{4}{|l|}{ Smoking status $\mathrm{n}$} \\
\hline Current smoker & 0 & 3 & 3 \\
\hline Past smoker & 7 & 13 & 8 \\
\hline Never-smoker & 4 & 0 & 0 \\
\hline Smoking history pack-years & $10.5 \pm 4.8$ & $72.7 \pm 14.1^{*}$ & $70.5 \pm 14.4^{*}$ \\
\hline $\mathrm{BMI} \mathrm{kg} \cdot \mathrm{m}^{-2}$ & $23.1 \pm 0.5$ & $21.6 \pm 0.9$ & $19.7 \pm 2.2$ \\
\hline FEV1/FVC & $81.7 \pm 2.1$ & $47.8 \pm 5.0 *$ & $41.2 \pm 3.5^{*}$ \\
\hline FEV 1 L & $2.55 \pm 0.11$ & $1.43 \pm 0.17^{*}$ & $1.06 \pm 0.14^{*}$ \\
\hline FEV $1 \%$ pred & $91.7 \pm 3.4$ & $54.7 \pm 5.5^{*}$ & $42.1 \pm 6.0^{*}$ \\
\hline FVC L & $3.14 \pm 0.14$ & $2.99 \pm 0.21$ & $2.59 \pm 0.23$ \\
\hline FVC $\%$ pred & $90.5 \pm 3.2$ & $84.7 \pm 7.4$ & $80.6 \pm 6.6$ \\
\hline \multicolumn{4}{|l|}{ COPD stage } \\
\hline I & & 2 & 1 \\
\hline ॥ & & 8 & 1 \\
\hline III & & 4 & 6 \\
\hline IV & & 2 & 3 \\
\hline Duration of COPD years & & $3.81 \pm 1.38$ & $4.72 \pm 1.09$ \\
\hline Peak work rate $\mathrm{W}$ & $93.2 \pm 8.4$ & $59.4 \pm 4.6^{*}$ & $72.3 \pm 7.0$ \\
\hline $\mathrm{PaO}_{2} \mathrm{mmHg}$ & & $79.0 \pm 3.4$ & $67.7 \pm 2.1^{* *}$ \\
\hline $\mathrm{PaCO}_{2} \mathrm{mmHg}$ & & $40.0 \pm 2.2$ & $40.5 \pm 1.8$ \\
\hline $\mathrm{SpO}_{2} \%$ at rest & $97.4 \pm 0.4$ & $96.1 \pm 0.4$ & $94.8 \pm 0.5^{*}$ \\
\hline
\end{tabular}

Data are presented as mean \pm SEM, unless otherwise stated. BMI: body mass index; COPD: chronic obstructive pulmonary disease; hypoxaemia: exertional hypoxaemia; FEV1: forced expiratory volume in $1 \mathrm{~s}$; FVC: forced vital capacity; \% pred: \% predicted; peak work rate: maximal incremental cycle work rate; $\mathrm{PaO}_{2}$ : arterial oxygen tension; $\mathrm{PaCO}_{2}$ : arterial carbon dioxide tension; $\mathrm{SpO}_{2}$ : arterial oxygen saturation measured by pulse oximetry. *: $p<0.05$ versus control; ${ }^{* *}$ : $p<0.05$ versus COPD without hypoxaemia.

Overall, average cortical oxy- $\mathrm{Hb}$ and deoxy- $\mathrm{Hb}$ concentrations were calculated in the pre-frontal area (figure $3 \mathrm{a}$ and $4 \mathrm{a}$ ), the left parietal area (figures $3 \mathrm{~b}$ and $4 \mathrm{~b}$ ) and the right parietal area (figures $3 \mathrm{c}$ and $4 \mathrm{c}$ ) during each minute of CWRE. Oxy-Hb concentrations (cortical oxygenation) of the pre-frontal area increased during exercise in the nonhypoxaemic group of patients with COPD and the control group (figure 3a). For hypoxaemic patients, however, oxy-Hb concentrations of the pre-frontal area showed a

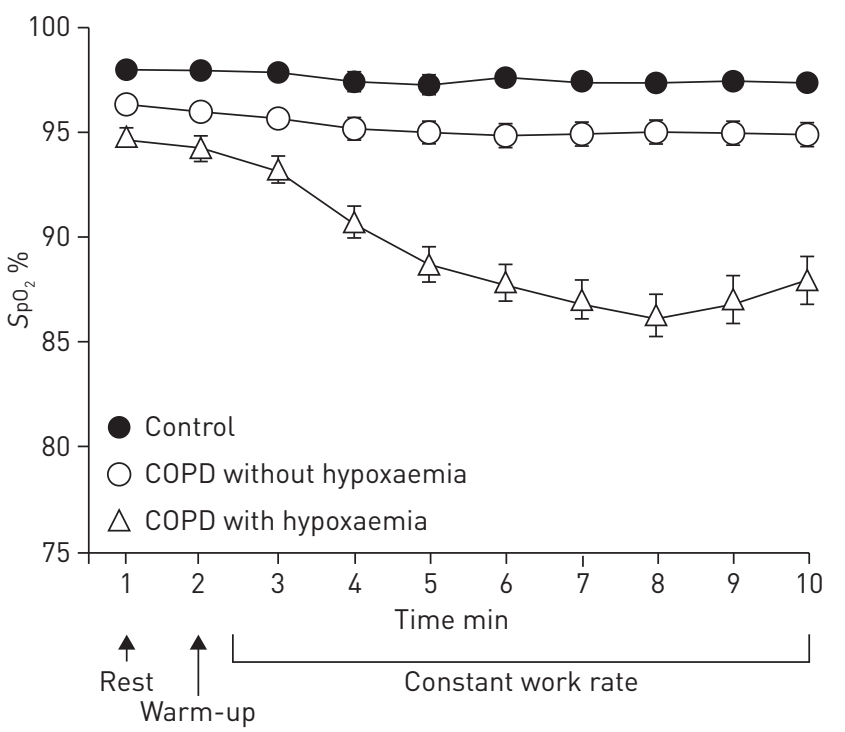

FIGURE 2 Time course of the changes in arterial oxygen saturation measured by pulse oximetry $\left(\mathrm{SpO}_{2}\right)$ in participants during low-intensity constant work rate cycling exercise. The data are presented as mean \pm SEM. COPD: chronic obstructive pulmonary disease. 


$$
\text { a) }
$$

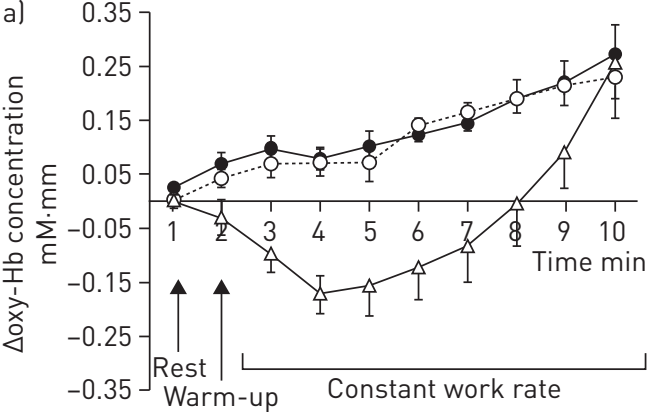

c)

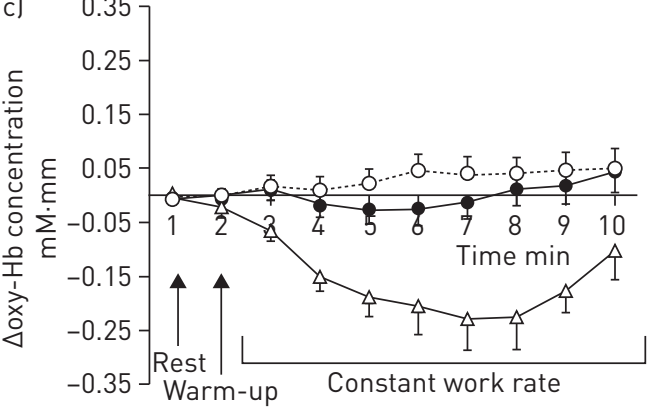

b) $\quad 0.35$

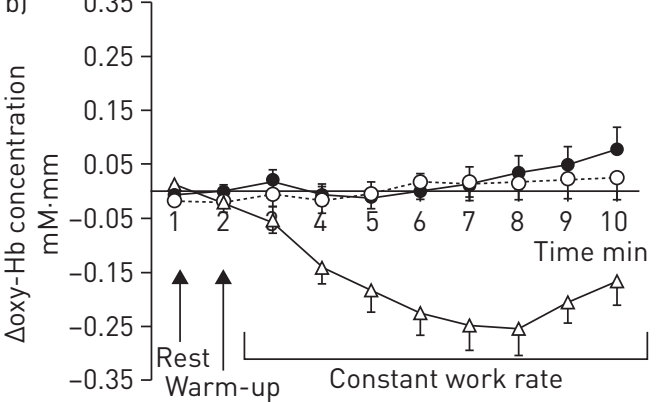

Control

COPD without hypoxaemia

$\triangle$ COPD with hypoxaemia

FIGURE 3 Time course of the changes in cortical oxyhaemoglobin loxy- $\mathrm{Hb}$ ) concentration in participants during low-intensity constant work rate cycling exercise. a) Pre-frontal probe, b) left parietal probe and c) right parietal probe. The data are presented as mean士SEM. COPD: chronic obstructive pulmonary disease.

biphasic response during exercise; oxy-Hb decreased during the early phase of exercise (1-5 min) and then increased during the later phase $(8-10 \mathrm{~min})$. From 2 to $9 \mathrm{~min}$, oxy-Hb concentrations of the pre-frontal area in the hypoxaemic group were significantly lower compared with the control and nonhypoxaemic COPD groups $(\mathrm{p}<0.05)$. Oxy-Hb concentrations of the bilateral parietal areas did not change during
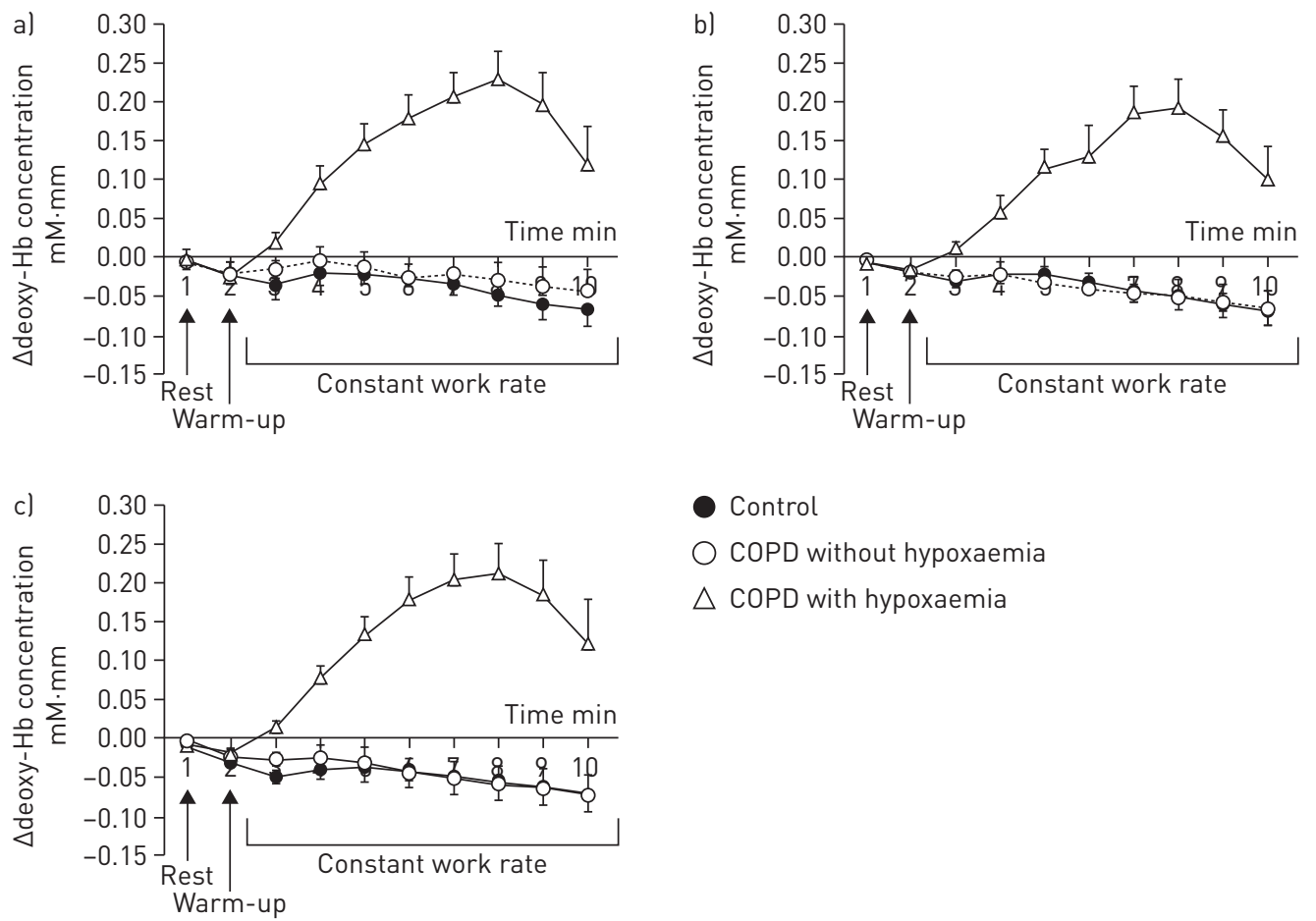

Control

COPD without hypoxaemia

$\triangle$ COPD with hypoxaemia

FIGURE 4 Time course of changes in cortical deoxyhaemoglobin (deoxy-Hb) concentration in participants during low-intensity constant work rate cycling exercise. a) Pre-frontal probe, b) left parietal probe and c) right parietal probe. The data are presented as mean士SEM. COPD: chronic obstructive pulmonary disease. 
exercise in the control and nonhypoxaemic groups, whereas they decreased in the hypoxaemic group. Deoxy-Hb concentrations of the pre-frontal and parietal areas decreased during exercise in the control and nonhypoxaemic groups (figure 4). However, deoxy-Hb of the pre-frontal and parietal areas increased in the hypoxaemic group during exercise and was maximal at $8 \mathrm{~min}$. Deoxy-Hb concentrations of the hypoxaemic group were significantly higher than seen in the control and nonhypoxaemic groups between 3 and $10 \mathrm{~min}$ as measured by the pre-frontal and parietal probes $(\mathrm{p}<0.05)$ (figure 4$)$. Total- $\mathrm{Hb}$ concentrations of the pre-frontal area increased during CWRE in all groups, but were unchanged in the right and left parietal areas (online supplementary figure S3). There were no significant differences in total-Hb concentrations among groups at any time assessed during the exercise period. In the hypoxaemic group, oxygen supplementation $\left(3 \mathrm{~L} \cdot \mathrm{min}^{-1}\right)$ resolved cortical underoxygenation (online supplementary figure S4).

\section{Parameters correlating with PDI based on the Borg scale}

Cortical activation was evaluated in eight cortical areas simultaneously to determine which area was most implicated in PDI in each group (figure 1). Based on an average of the last 4 min of exercise, none of the ventilatory parameters (oxygen uptake $\left(V^{\prime} \mathrm{O}_{2}\right)$, carbon dioxide production $\left(V^{\prime} \mathrm{CO}_{2}\right)$, minute ventilation $\left(V^{\prime} \mathrm{E}\right)$, dead space volume $(V \mathrm{D}) / V \mathrm{~T}$, mean inspiratory flow $(V \mathrm{~T} /$ inspiratory time $(t \mathrm{I})$ ), inspiratory duty cycle $(t \mathrm{t} / \mathrm{tTOTAL}), \mathrm{PETCO}_{2}$, end-tidal oxygen tension $\left(P_{\mathrm{ETO}_{2}}\right)$, respiration rate and $\left.V \mathrm{~T}\right)$ were correlated with PDI (online supplementary table S2). PDI was not correlated with $\mathrm{SpO}_{2}$, systolic blood pressure, diastolic blood pressure or heart rate. Although $\mathrm{PETCO}_{2}$ is known to affect cortical blood flow, there was no correlation between the deoxy- $\mathrm{Hb}$ concentration of any cortex and $\mathrm{PETCO}_{2}$ in our analysis, except for the $\mathrm{M} 1$ area in COPD patients without hypoxaemia (online supplementary table S3). PDI was negatively correlated with changes in deoxy- $\mathrm{Hb}$ in the right PMA and left $\mathrm{S} 1$ in the control participants (table 2 and figure 5). In the nonhypoxaemic COPD participants, PDI was negatively correlated with deoxy-Hb in the left PMA. These results indicate that PMA activation was correlated with PDI in the control participants and nonhypoxaemic COPD patients. In the hypoxaemic patients, PDI was positively correlated with deoxy- $\mathrm{Hb}$ in the following regions: orbitofrontal, dorsolateral, left and right $\mathrm{M} 1$, and right S1. Under oxygen supplementation, deoxy-Hb in the PMA was negatively correlated with PDI in the hypoxaemic COPD patients, as in the nonhypoxaemic COPD patients. These results indicate that deoxygenation of the cortex is correlated with PDI in hypoxaemic COPD patients. In all groups, the average total-Hb concentrations, which reflect cortical blood flow and $\mathrm{SpO}_{2}$, were not correlated with PDI.

\section{Discussion}

This study examined the cortical haemodynamics of patients with COPD with and without exertional hypoxaemia. Cortical oxygenation was impaired in hypoxaemic patients compared with control participants and nonhypoxaemic patients, as confirmed by the decrease in oxy-Hb and the increase in deoxy-Hb. Cortical deoxygenation was ameliorated by oxygen supplementation. JENSEN et al. [6] also reported exercise-induced

TABLE 2 Correlation ( $\rho$ ) between perceived dyspnoea intensity based on the Borg score [22] with concentration changes in deoxyhaemoglobin during low-intensity constant work rate cycling exercise

\begin{tabular}{lcccc} 
Cortical area & Control $^{\#}$ & \multicolumn{2}{c}{ COPD } \\
\cline { 3 - 4 } & & Without hypoxaemia" & \multicolumn{2}{c}{ With hypoxaemia } \\
\cline { 3 - 4 } & & & Room air & Plus oxygen \\
\hline Dorsolateral & -0.33 & -0.02 & $0.75^{*}$ & -0.01 \\
Orbitofrontal & 0.04 & 0.05 & $0.66^{*}$ & -0.11 \\
Left PMA & -0.33 & $-0.51^{*}$ & 0.39 & -0.22 \\
Left M1 & $-0.75^{*}$ & -0.08 & $0.71^{*}$ & -0.27 \\
Left S1 & 0.01 & -0.00 & $0.59^{*}$ & -0.11 \\
Right PMA & $-0.84^{*}$ & -0.20 & 0.27 & $-0.66^{*}$ \\
Right M1 & -0.33 & 0.14 & 0.47 & $-0.68^{*}$ \\
Right S1 & -0.53 & -0.13 & 0.65 & -0.16 \\
\hline
\end{tabular}

COPD: chronic obstructive pulmonary disease; hypoxaemia: exertional hypoxaemia; PMA: pre-motor cortex; M1: primary motor cortex; S1: primary somatosensory cortex. ${ }^{*}$ : $p<0.05$ for correlation with PDI. A negative coefficient indicates dyspnoea intensity is correlated with decreased deoxy-Hb concentration, which reflects cortical activation. In contrast, a positive coefficient indicates dyspnoea intensity is correlated with increased deoxy- $\mathrm{Hb}$ concentration, which reflects impaired oxygenation of the cortex. ${ }^{\#}: \mathrm{n}=11 ;{ }^{\text {ๆ }} \mathrm{n}=16$. 

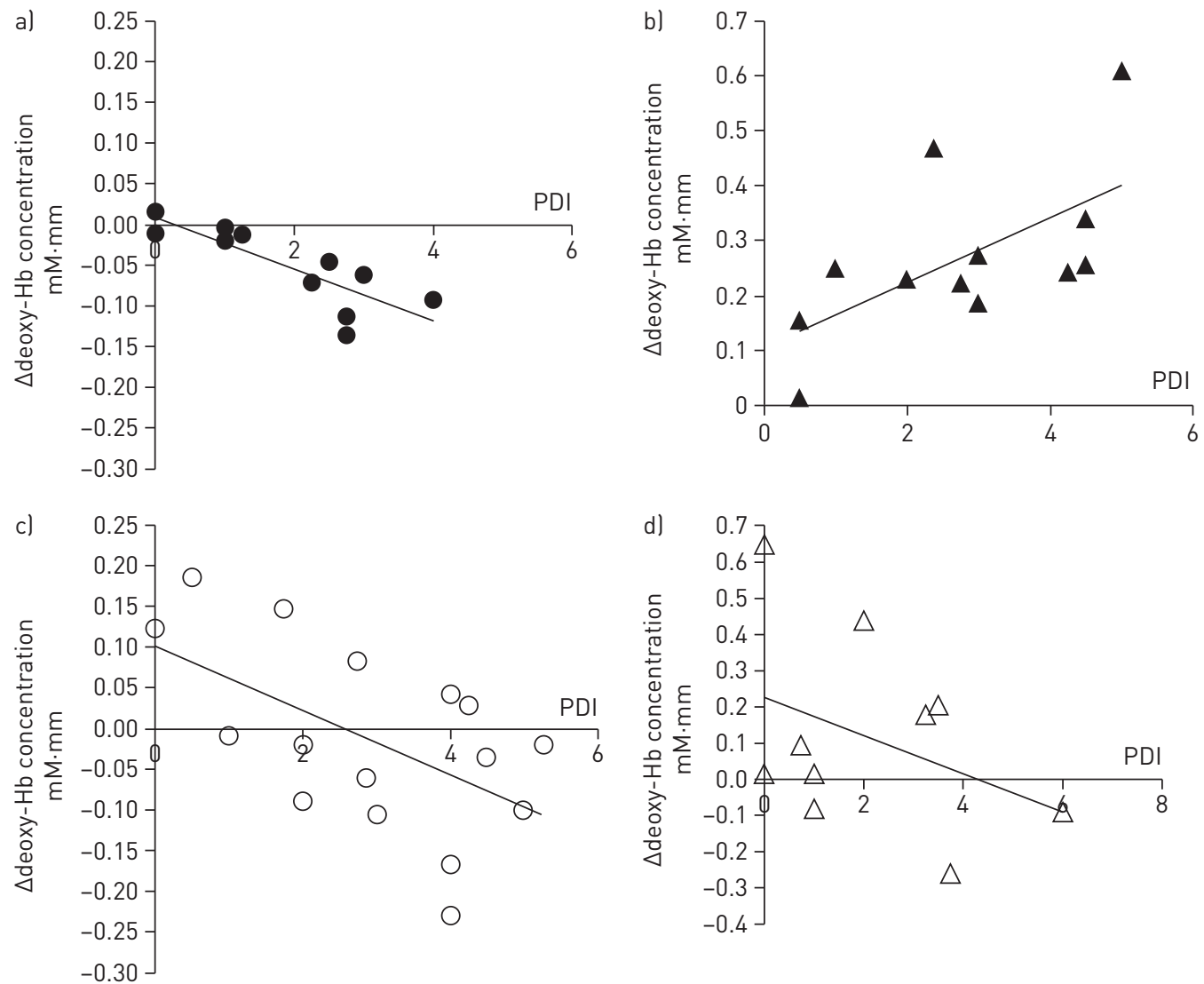

FIGURE 5 Correlation between perceived dyspnoea intensity (PDI) (Borg scale rating) and cortical deoxyhaemoglobin (deoxy-Hb) concentration in participants in the a) control, b) deoxy-Hb without hypoxaemia, c) chronic obstructive pulmonary disease (COPD) with hypoxaemia and d) COPD with hypoxaemia under oxygen supplementation groups. PDI and cortical deoxy- $\mathrm{Hb}$ concentration are average values of the last 4 min of exercise. PDI was correlated with decreased deoxy-Hb concentration in the right pre-motor cortex (PMA) of the a) control group $(\rho=-0.84, p<0.01)$ and left PMA in the b) COPD without hypoxaemia group $(\rho=-0.51$, $\mathrm{p}<0.05)$. c) In contrast. PDI was correlated with increased dorsolateral area deoxy- $\mathrm{Hb}$ concentration in the COPD with hypoxaemia group $(\rho=0.75, p<0.05)$. d) Under oxygen supplementation, PDI was correlated with decreased deoxy- $\mathrm{Hb}$ concentration in the right PMA in the hypoxaemic COPD group $(p=-0.66, p<0.05)$.

cortical deoxygenation (increased deoxy-Hb) in patients with terminal lung disease, but oxy-Hb did not decrease during exercise. There were some methodological differences between our study and that of JENSEN et al. [6]. Hypoxaemic patients were not compared with nonhypoxaemic patients or control participants by JENSEN et al. [6], precluding a demonstration of the decline in oxy-Hb. Furthermore, cortical haemodynamics were examined via a single-channel NIRS probe on the pre-frontal area during incremental exercise, whereas we used multichannel NIRS probes on the pre-frontal area and on the left and right parietal area simultaneously, permitting us to more globally measure cortex haemodynamics. We revealed that in all groups the cortical blood flow in the pre-frontal area increased, but not in the parietal areas during CWRE. Most previous reports have also shown that cortical oxygenation (oxy-Hb) and blood flow (total-Hb) in the pre-frontal area increased in response to an increase in exercise [12, 14]. SubUDHI et al. [13] showed that cerebral oxy- $\mathrm{Hb}$ increased during low-to-middle intensity exercise $(25-75 \%$ of peak) and decreased during peak exercise as a result of decreased partial pressure of carbon dioxide $\left(\mathrm{PaCO}_{2}\right)$. Hyperventilation during peak exercise decreases $\mathrm{PaCO}_{2}$, which can result in cerebral vasoconstriction and diminished cerebral blood flow. BhambHani et al. [24] showed that $\mathrm{PETCO}_{2}$ increased from the beginning of exercise and then began to decline at the threshold of respiratory compensation, and that cortical blood flow measured by NIRS also changed in parallel with $\mathrm{PETCO}_{2}$ during incremental exercise. Their results affected our decision to minimise the change in $\mathrm{PETCO}_{2}$, by assessing participants using mild constant work exercise instead of incremental exercise. Actually, $\mathrm{PETCO}_{2}$ was not correlated with deoxy-Hb for the average the of measurements obtained during the last $4 \mathrm{~min}$ of exercise. $\mathrm{PETCO}_{2}$ was increased only during the first $4 \mathrm{~min}$ of exercise, including the rest and warm-up periods, and did not change during exercise; therefore, cortical haemodynamics might not have been altered. However, we could not completely avoid the effects of $\mathrm{PETCO}_{2}$ while studying cortical hemodynamics during exercise. 
PDI during exercise was significantly correlated with cortical activation of the PMA areas in control participants and nonhypoxaemic patients with COPD. This result is consistent with a recent report on the corticolimbic neural responses to air hunger [25]. BINKs et al. [25] showed that cortical activity of PMA was correlated with a sustained strong sensation of dyspnoea (air hunger), while the neural activity of the anterior cingulate cortex, pre-supplementary motor area and middle frontal gyri were correlated with mild dyspnoea in healthy subjects. We previously reported that activation of the pre-frontal cortex was correlated with exertional dyspnoea [12]. Nyвo and NiELSEN [26] also showed that perceived exertion was associated with pre-frontal cortex activity in healthy participants. In this study, we specifically analysed the activation area and found that PMA activation had a stronger correlation with exertional dyspnoea compared with the pre-frontal cortex area. In the pre-frontal area, oxy- $\mathrm{Hb}$ and deoxy-Hb may be influenced by the increase in total cortical blood flow (total-Hb) during exercise. In contrast, the overall concentration of total- $\mathrm{Hb}$ did not increase in the parietal area, including the PMA, during exercise; thus, the change in deoxy-Hb in the PMA area may be more indicative of cortical activation.

Little is known about the afferent pathways responsible for dyspnoea [27]. Since dyspnoea involves several distinct types of sensation, it would be expected that the afferent mechanisms responsible for dyspnoea are complicated. Two major pathways have been postulated to be involved in sending signals associated with respiratory sensations to the cortex $[9,27]$. The first pathway arises predominantly from respiratory muscle afferents, relayed in the brainstem medulla and projected to the ventroposterior thalamus area, from where thalamocortical projections ascend to the primary and secondary somatosensory cortex. The second pathway includes mainly vagal afferents from the lungs and airways, which are relayed in the brainstem medulla, and projections ascend to the amygdala, medial dorsal areas of the thalamus and the insula cortex. This predominantly limbic pathway might further include the operculum and other pre-frontal areas, and might be more strongly associated with the affective components influencing the experience of breathlessness. Both pathways include final projections to the higher motor cortex, including the M1, PMA and supplementary motor area, whereas efferent motor commands are projected to respiratory muscles. We could not determine which pathway was predominantly responsible for the PMA activation observed in this study, but both pathways may have contributed to cortical activation.

In hypoxaemic patients with COPD, PDI is correlated with increased cortical deoxy-Hb during exercise, but not $\mathrm{SpO}_{2}$. The increases in deoxy-Hb concentration and PDI both peaked at $8 \mathrm{~min}$ (online supplementary figure S6). Therefore, cortical deoxygenation may have a stronger correlation with the perceptions of dyspnoea than desaturation in these patients. Oxygen supplementation changed the relationship between PDI and cortical deoxy-Hb in these patients. PDI was correlated with decreased deoxy-Hb in the PMA, which reflects cortical activation, as in nonhypoxaemic COPD patients. Oxygen supplementation may wash out the strong effect of desaturation and the hidden cortical response to PDI may appear.

The global increase in cerebral blood flow in response to hypoxia is due both to the direct vascular effect of hypoxia on the endothelium and to cerebral vasodilation mediated by vasodilator metabolites [28]. PAGANI et al. [29] showed that the changes in regional cerebral blood flow ( $\mathrm{rCBF}$ ) in response to hypoxia are different in the gray and white matter. They found a greater degree of $\mathrm{rCBF}$ in the forebrain grey matter during acute hypoxia and speculated that this localised response might be ascribed to an acute compensatory mechanism involving regions with increased sensitivity to oxygen deficits, possibly because they would develop reversible neuronal impairment. However, Dyer et al. [30] found that there was no difference in the percentage change of $\mathrm{rCBF}$ in different regions of the brain during acute hypoxia. The discrepant findings might have been due to differences in the degree of hypoxia or differences in the methods used to assess rCBF (fMRI versus single-photon emission computed tomography). In this study, the total-Hb concentration, which reflects regional perfusion, increased in the pre-frontal cortex during exercise and the increase was larger in COPD patients with hypoxia compared with other groups (online supplementary figure S3). However, the difference was not statistically significant, because of considerable variation between patients.

Oxygen supplementation improved exertional desaturation for all patients, but only six of 11 hypoxaemic COPD patients achieved improvement in dyspnoea. Oxygen supplementation may not be sufficient for improving the dyspnoea of some patients, although supplementation was sufficient for maintaining $\mathrm{SpO}_{2}$. A meta-analysis found that the use of oxygen relieves exertional dyspnoea [4], but not for every patient. $\mathrm{SpO}_{2}$ or arterial oxygen tension $\left(\mathrm{PaO}_{2}\right)$ measurements may not be sufficient for adjusting the flow of oxygen to provide symptom relief. Cortical oxygenation measurements may be needed for adjusting the volume of oxygen supplementation during exercise. Further study is needed for clarification.

In recent years, methods such as blood oxygen level-dependent fMRI (BOLD-fMRI) or positron emission tomography (PET) of the brain have been developed to reveal the activity of different brain regions during various cognitive or motor tasks. BINKs et al. [25] recently reported on the good temporal resolution of BOLD signals that were related to air hunger in healthy study participants with mild hypercapnia. They 
found that two distinct regions were activated and manifested distinct temporal profiles after the onset of air hunger. However, both BOLD-fMRI and PET require complete immobilisation of the head and are therefore unsuitable for the assessment of exercising COPD patients. NIRS is a more practical method and has been used to assess the brain activity of patients performing exercise $[14,31]$. NIRS oxy-Hb and deoxy-Hb signals are strongly correlated with BOLD-fMRI signals [32]. As patients with COPD usually perceive dyspnoea during exercise, but not at rest, NIRS is better for identifying the cortical activation associated with the exertional dyspnoea of these patients [31].

Dyspnoea is the result of a dissociation or mismatch between the degree of central ventilatory drive and the magnitude of respiratory effort [27]. In other words, dyspnoea is the result of dissociation between outgoing motor signals to the respiratory muscles and incoming afferent information. PMA activation possibly reflects increased ventilatory drive, but not the production of PDI. We used low-intensity CWRE as the dyspnogenic stimulus. The exercise load by itself possibly led to a change in cortical hemodynamics and induced the activation of PMA, although we chose to use constant low-intensity exercise to minimise the effect of exercise on cortical activation. Using NIRS assessment, ALEXANDRE et al. [31] reported that there was cortical activation of the PMA, MI and S1 regions of COPD patients performing submaximal exercise of their knee extensors. Goswami et al. [33] reported that electrical stimulation of the wrist flexors at a submotor threshold increased neural activity in the bilateral posterior insula, ventral medial pre-frontal cortex, subgenualanterior cingulate cortex, mid-cingulate cortex and posterior cingulate cortex. Therefore, we cannot rule out the effect of skeletal muscle afferent activity on cortical activation. MAEKURA et al. [34] found that the arterial $\mathrm{pH}$ decreased rapidly in COPD patients with severely reduced exercise capacity, and that their acidaemia was a result of both lactic acidosis and respiratory acidosis. We did not measure arterial lactic acid in this study, but increases in arterial lactic acid levels might have been different in the different groups of patients and might have resulted in different effects on cerebral metabolism or oxygen uptake [14].

There are limitations to this study. NIRS does not measure the absolute blood flow or absolute oxygenation of the cortex; rather, it measures changes from baseline. Therefore, it is a method best suited for within-subject comparisons. We could not investigate whole-brain activity because NIRS is limited to the detection of cortical haemodynamics. As PET and fMRI are excellent for spatial analysis, these methods are ideal for localising brain activity. By comparison, NIRS is an excellent tool for temporal analysis; it allowed us to easily monitor brain activity during exercise without restrictions.

Our results cannot provide a direct correlation between PMA activation and exertional dyspnoea in patients with COPD. Our results indicate that cortical activation may be correlated with increased respiratory motor drive. Using PET scanning of healthy study participants, FINK et al. [35] showed that cortical activation of PMA, M1 and the somatosensory area was correlated with ventilatory motor drive. The involvement of these same cortical areas reflects our findings in participants with exertional dyspnoea. Further study is needed to clarify the mechanisms involved in the activation of the PMA and exertional dyspnoea in COPD patients.

In conclusion, our study used NIRS to demonstrate that cortical oxygenation was impaired in hypoxaemic patients with COPD during exercise, compared with control participants and nonhypoxaemic patients. Exertional dyspnoea was associated with activation of the motor cortex in nonhypoxaemic and control participants, whereas it was associated with impaired oxygenation of the cortex in hypoxaemic patients.

\section{References}

1 Casanova C, Cote C, Marin JM, et al. Distance and oxygen desaturation during the 6-min walk test as predictors of long-term mortality in patients with COPD. Chest 2008; 134: 746-752.

2 Panos RJ, Eschenbacher W. Exertional desaturation in patients with chronic obstructive pulmonary disease. COPD 2009; 6: 478-487.

3 Moore RP, Berlowitz DJ, Denehy L, et al. A randomised trial of domiciliary, ambulatory oxygen in patients with COPD and dyspnoea but without resting hypoxaemia. Thorax 2011; 66: 32-37.

4 Uronis H, McCrory DC, Samsa G, et al. Symptomatic oxygen for non-hypoxaemic chronic obstructive pulmonary disease. Cochrane Database Syst Rev 2011: CD006429.

5 Dodd JW, Getov SV, Jones PW. Cognitive function in COPD. Eur Respir J 2010; 35: 913-922.

6 Jensen G, Nielsen HB, Ide K, et al. Cerebral oxygenation during exercise in patients with terminal lung disease. Chest 2002; 122: 445-450.

7 Oliveira MF, Rodrigues MK, Treptow E, et al. Effects of oxygen supplementation on cerebral oxygenation during exercise in chronic obstructive pulmonary disease patients not entitled to long-term oxygen therapy. Clin Physiol Funct Imaging 2012; 32: 52-58.

8 Parshall MB, Schwartzstein RM, Adams L, et al. An Official American Thoracic Society statement: update on the mechanisms, assessment, and management of dyspnea. Am J Respir Crit Care Med 2012; 185: 435-452.

9 von Leupoldt A, Dahme B. Cortical substrates for the perception of dyspnea. Chest 2005; 128: 345-354

10 Evans KC, Banzett RB, Adams L, et al. BOLD fMRI identifies limbic, paralimbic, and cerebellar activation during air hunger. J Neurophysiol 2002; 88: 1500-1511. 
11 von Leupoldt A, Sommer T, Kegat S, et al. The unpleasantness of perceived dyspnea is processed in the anterior insula and amygdala. Am J Respir Crit Care Med 2008; 177: 1026-1032.

12 Higashimoto $\mathrm{Y}$, Honda N, Yamagata $\mathrm{T}$, et al. Activation of the prefrontal cortex is associated with exertional dyspnea in chronic obstructive pulmonary disease. Respiration 2011; 82: 492-500.

13 Subudhi AW, Dimmen AC, Roach RC. Effects of acute hypoxia on cerebral and muscle oxygenation during incremental exercise. J Appl Physiol 2007; 103: 177-183.

14 Ide K, Secher NH. Cerebral blood flow and metabolism during exercise. Prog Neurobiol 2000; 61: 397-414

15 Gervain J, Macagno F, Cogoi S, et al. The neonate brain detects speech structure. Proc Natl Acad Sci USA 2008; 105: 14222-14227.

16 Pena M, Maki A, Kovacic D, et al. Sounds and silence: an optical topography study of language recognition at birth. Proc Natl Acad Sci U S A 2003; 100: 11702-11705.

17 Kobayashi E, Kusaka T, Karaki M, et al. Functional optical hemodynamic imaging of the olfactory cortex. Laryngoscope 2007; 117: 541-546.

18 Taga G, Asakawa K, Maki A, et al. Brain imaging in awake infants by near-infrared optical topography. Proc Natl Acad Sci U S A 2003; 100: 10722-10727.

19 Okamoto M, Dan H, Sakamoto K, et al. Three-dimensional probabilistic anatomical cranio-cerebral correlation via the international 10-20 system oriented for transcranial functional brain mapping. Neuroimage 2004; 21: $99-111$.

20 Okamoto M, Dan I. Automated cortical projection of head-surface locations for transcranial functional brain mapping. Neuroimage 2005; 26: 18-28.

21 Shibuya K, Ueda C, Sato K, et al. Perceived exertion is not necessarily associated with altered brain activity during exercise. J Physiol Anthropol 2009; 28: 63-69.

22 Borg GA. Psychophysical bases of perceived exertion. Med Sci Sports Exerc 1982; 14: 377-381.

23 Puente-Maestu L, Garcia de Pedro J, Martinez-Abad Y, et al. Dyspnea, ventilatory pattern, and changes in dynamic hyperinflation related to the intensity of constant work rate exercise in COPD. Chest 2005; 128: 651-656.

24 Bhambhani Y, Malik R, Mookerjee S. Cerebral oxygenation declines at exercise intensities above the respiratory compensation threshold. Respir Physiol Neurobiol 2007; 156: 196-202.

25 Binks AP, Evans KC, Reed JD, et al. The time-course of cortico-limbic neural responses to air hunger. Respir Physiol Neurobiol 2014; 204: 78-85.

26 Nybo L, Nielsen B. Perceived exertion is associated with an altered brain activity during exercise with progressive hyperthermia. J Appl Physiol 2001; 91: 2017-2023.

27 Nishino T. Dyspnoea: underlying mechanisms and treatment. Br J Anaesth 2011; 106: 463-474.

28 Pearce WJ. Mechanisms of hypoxic cerebral vasodilatation. Pharmacol Ther 1995; 65: 75-91.

29 Pagani M, Salmaso D, Sidiras GG, et al. Impact of acute hypobaric hypoxia on blood flow distribution in brain. Acta Physiol (Oxf) 2011; 202: 203-209.

30 Dyer EA, Hopkins SR, Perthen JE, et al. Regional cerebral blood flow during acute hypoxia in individuals susceptible to acute mountain sickness. Respir Physiol Neurobiol 2008; 160: 267-276.

31 Alexandre F, Heraud N, Oliver N, et al. Cortical implication in lower voluntary muscle force production in non-hypoxemic COPD patients. PLoS One 2014; 9: e100961.

32 Strangman G, Culver JP, Thompson JH, et al. A quantitative comparison of simultaneous BOLD fMRI and NIRS recordings during functional brain activation. Neuroimage 2002; 17: 719-731.

33 Goswami R, Frances MF, Shoemaker JK. Representation of somatosensory inputs within the cortical autonomic network. Neuroimage 2011; 54: 1211-1220.

34 Maekura R, Hiraga T, Miki K, et al. Differences in physiological response to exercise in patients with different COPD severity. Respir Care 2014; 59: 252-262.

35 Fink GR, Corfield DR, Murphy K, et al. Human cerebral activity with increasing inspiratory force: a study using positron emission tomography. J Appl Physiol 1996; 81: 1295-1305. 